\title{
Layered hydroxide anion exchanger and their applications related to pesticides: a brief review
}

\begin{abstract}
Layered double hydroxides and layered hydroxide salts have generated enormous excitement in the inorganic field due to their potential to act as versatile host materials in fabricating novel host-guest layered materials. The ability of the layered hydroxide anion exchanger to be incorporated with a wide range of guest ions enable them to be exploited in various applications related to pesticides. This review sums up the different methods of preparing layered hydroxide anion exchanger, summarises the types of anion intercalated into these layered hydroxide anion exchanger based on their respective systems, and elucidates their potential applications in pesticide-related fields.
\end{abstract}

Keyword:Layered double hydroxides; Layered hydroxide salts; Hydrotalcite; Nanocomposites; Pesticides 